ARTICLE

Received 30 Aug 2014 | Accepted 14 Jan 2015 | Published 18 Feb 2015 DOl: 10.1038/ncomms7311

\title{
Tetrahedrally coordinated carbonates in Earth's lower mantle
}

\author{
Eglantine Boulard ${ }^{1, \star}, \dagger$, Ding Pan ${ }^{2, \star}$, Giulia Galli², Zhenxian Liu $^{3} \&$ Wendy L. Mao ${ }^{1,4}$
}

Carbonates are the main species that bring carbon deep into our planet through subduction. They are an important rock-forming mineral group, fundamentally distinct from silicates in the Earth's crust in that carbon binds to three oxygen atoms, while silicon is bonded to four oxygens. Here we present experimental evidence that under the sufficiently high pressures and high temperatures existing in the lower mantle, ferromagnesian carbonates transform to a phase with tetrahedrally coordinated carbons. Above $80 \mathrm{GPa}$, in situ synchrotron infrared experiments show the unequivocal spectroscopic signature of the high-pressure phase of $(\mathrm{Mg}, \mathrm{Fe}) \mathrm{CO}_{3}$. Using ab-initio calculations, we assign the new infrared signature to $\mathrm{C}-\mathrm{O}$ bands associated with tetrahedrally coordinated carbon with asymmetric $\mathrm{C}-\mathrm{O}$ bonds. Tetrahedrally coordinated carbonates are expected to exhibit substantially different reactivity than low-pressure threefold coordinated carbonates, as well as different chemical properties in the liquid state. Hence, this may have significant implications for carbon reservoirs and fluxes, and the global geodynamic carbon cycle.

\footnotetext{
${ }^{1}$ Geological and Environmental Sciences, Stanford University, Stanford, California 94305, USA. ${ }^{2}$ Institute for Molecular Engineering, University of Chicago, Chicago, Illinois 60637, USA. ${ }^{3}$ Geophysical Laboratory, Carnegie Institution of Washington, Washington, District Of Columbia 20015 , USA. ${ }^{4}$ Photon Science, SLAC National Accelerator Laboratory, Menlo Park, California 94025, USA. ${ }^{*}$ These authors contributed equally to this work. $\dagger$ Present address: Institut NEEL, CNRS, 25 Rue des Martyrs, 38042 Grenoble, France. Correspondence and requests for materials should be addressed to E.B. (email: eglantine.boulard@neel.cnrs.fr).
} 
F erromagnesite $\left((\mathrm{Mg}, \mathrm{Fe}) \mathrm{CO}_{3}\right)$ minerals are probable candidates for deep-Earth carbon storage and are expected to play a key role in the deep carbon cycle (for example, see refs 1,2). Therefore, the properties of ferromagnesite at lower mantle conditions have been the focus of many theoretical and experimental studies ${ }^{2-8}$. Results of high-pressure experiments have suggested that the rhombohedral structure of magnesite $\left(\mathrm{MgCO}_{3}\right)$ is stable up to $115 \mathrm{GPa}$ at $2,000-3,000 \mathrm{~K}$, and that it transforms to a new structure at higher pressures ${ }^{2}$. On the other hand, first-principles calculations predict that magnesite transforms into a phase containing $\mathrm{CO}_{4}$ tetrahedral groups at lower pressure $(\sim 82 \mathrm{GPa})^{5}$. Boulard et al. ${ }^{6}$ proposed that magnesite and ferromagnesite adopt the theoretically proposed structure above $80 \mathrm{GPa}$ at $2,300 \mathrm{~K}$, based on in situ X-ray diffraction (XRD) and ex situ spectroscopy analysis on recovered samples (electron energy loss spectroscopy and scanning transmission X-ray microscopy performed at the $\mathrm{C}-\mathrm{K}$ edge). However, these observations have been controversial for two reasons: (1) only LeBail refinements were performed on the highpressure, high-temperature XRD data and, hence, determination of the atomic positions within the unit cell was not possible; (2) spectroscopic analyses were performed ex situ on recovered samples after temperature quenching and decompression to ambient conditions, where transmission electron microscopic analyses showed amorphization of the sample indicating the high-pressure crystal structure was not preserved.

Here we report the first unequivocal evidence of tetrahedrally coordinated carbon in high-pressure carbonates, obtained by a combined experimental and theoretical study. We perform in situ synchrotron infrared spectroscopic studies on ferromagnesite in diamond anvil cells (DAC) and identify a unique vibrational signature present only in the high-pressure phase. We perform $a b$ initio calculations of the infrared spectra, which allow us to assign this vibrational signature to asymmetric, $\mathrm{sp}^{3}$-like $\mathrm{C}-\mathrm{O}$ bonds.

\section{Results}

In situ infrared spectroscopy characterization. Magnesite and siderite $\left(\mathrm{FeCO}_{3}\right)$ form a solid solution at ambient conditions and adopt the same structure at high pressure and temperature, except that on substitution of $\mathrm{Mg}$ by $\mathrm{Fe}$, the volume of the unit cell decreases by about $7 \%$ (ref. 6) $\left(\mathrm{V}\left(\mathrm{MgCO}_{3}\right)=351.7 \AA^{3}\right.$ at $85 \mathrm{GPa}$ and $2,400 \pm 150 \mathrm{~K}$ and $\mathrm{V}\left(\mathrm{Mg}_{0.25} ; \mathrm{Fe}_{0.75} \mathrm{CO}_{3}\right)=328.9 \AA^{3}$ at $80 \mathrm{GPa}$ and $300 \mathrm{~K}$ ). We note that only the Fe-bearing phase is temperature quenchable $^{6}$, an important requirement for infrared spectra measurements in the DAC. We thus chose a natural sample of ferromagnesite with a composition $\left(\mathrm{Mg}_{0.25} \mathrm{Fe}_{0.75}\right) \mathrm{CO}_{3}$, which was measured with an electron microprobe. Here we refer to the high-

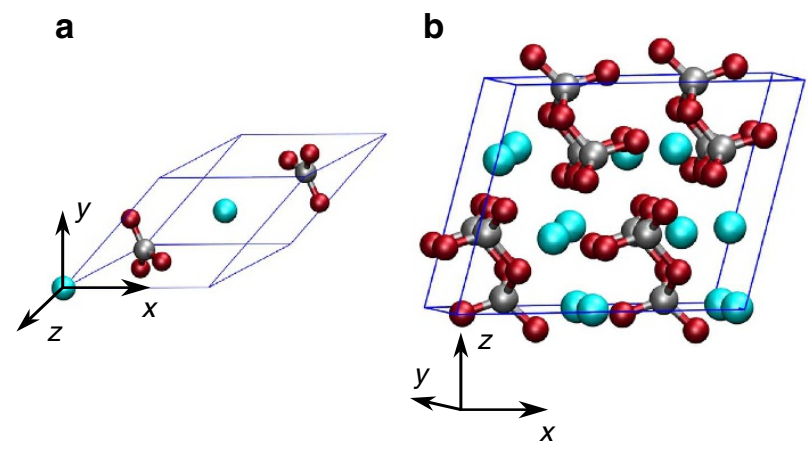

Figure 1 | Ball and stick representation of the two structures.

Ferromagnesite at ambient conditions (a) and the post-magnesite phase above $80 \mathrm{GPa}$ (b). Red, grey and blue spheres represent oxygen, carbon and cations ( $\mathrm{Fe}$ or $\mathrm{Mg}$ ) atoms, respectively. pressure structure of ferromagnesite as post magnesite. In situ infrared experiments were performed at the National Synchrotron Light Source on the high-pressure infrared beamline U2A. Two experiments were conducted: (1) infrared spectra were collected on compression and decompression of $\left(\mathrm{Mg}_{0.25} \mathrm{Fe}_{0.75}\right) \mathrm{CO}_{3}$ between 0 and $54 \mathrm{GPa}$ at ambient temperature. As the sample was not heated, $\left(\mathrm{Mg}_{0.25} \mathrm{Fe}_{0.75}\right) \mathrm{CO}_{3}$ did not transform into the post-magnesite structure, but only experienced an isostructural electronic spin transition around $50 \mathrm{GPa}^{9,10}$. (2) In a second set of experiments, powdered $\left(\mathrm{Mg}_{0.25} \mathrm{Fe}_{0.75}\right) \mathrm{CO}_{3}$ was compressed up to $103 \mathrm{GPa}$ and then transformed into the post-magnesite phase when laser heated at $\sim 2,100 \mathrm{~K}$. In situ XRD was used to monitor the transformation into the post-magnesite phase (Supplementary Fig. 1). Infrared spectra were recorded at the highest pressure of $103 \mathrm{GPa}$ and during decompression of the post-magnesite phase back to $0 \mathrm{GPa}$ at room temperature.

At low pressure, ferromagnesite is composed of repeating, alternating layers of $\left(\mathrm{CO}_{3}\right)^{2-}$ radicals (anions) and layers of divalent, positively charged cations $\left(\mathrm{Fe}^{2+}\right.$ and $\left.\mathrm{Mg}^{2+}\right)$ (Fig. 1a). The mid-infrared spectral absorption features result primarily from fundamental internal vibrations of the $\mathrm{C}-\mathrm{O}$ bonds in the carbonate radical: the out-of-plane bending $\left(v_{2}\right)$, the asymmetric stretch $\left(v_{3}\right)$ and in-plane bending $\left(v_{4}\right)$ modes (for example see, ref. 11). At ambient conditions, we measured these three modes on the polycrystalline carbonate phase (symmetry group: R-3C) at $867,1,460$ and $739 \mathrm{~cm}^{-1}$, respectively (Fig. 2a). Frequency of $v_{3}$ was determined using a thinner sample (cf. Supplementary Fig. 2). These frequencies are in good agreement with those reported by previous infrared studies on iron-bearing carbonates $3,11,12$. Additional modes resulting from the combination of the three fundamental ones are also present: a band at $1,811 \mathrm{~cm}^{-1}$, which stems from the combination of $v_{1}+v_{4}$, and one at $2,512 \mathrm{~cm}^{-1}$, which corresponds to the combination $2 v_{2}+v_{3}$ (ref. 13). Under compression at room temperature, no additional infrared bands were observed; rather, a shift to higher wave numbers was detected for all infrared bands, except for the transverse-optical (TO) component of the $v_{2}$ band, which exhibited a slight negative pressure shift $\left(-0.29 \mathrm{~cm}^{-1} \mathrm{GPa}^{-1}\right)$. Such a shift is in agreement with previous studies on iron-bearing carbonates ${ }^{3}$ and has been interpreted as stemming from the increased strength of the divalent cation-oxygen bonds under compression. We observed no hysteresis on decompression of the untransformed carbonate.

In the second set of experiments, several new infrared bands were observed after transformation into the post-magnesite phase by laser heating (Fig. 2a). At very high pressure (from 103 to $81 \mathrm{GPa}$ ), the most intense infrared bands appeared to be saturated, which made their positions difficult to determine precisely; however, our experiment showed that between 103 and $43 \mathrm{GPa}$ these new infrared modes gradually shift to lower wavenumbers (Fig. 2b). At $0 \mathrm{GPa}$, the experimental spectrum does not correspond to a carbonate phase and displayed infrared modes at $746,836,872,1,092,1,480,1,823,2,349$ and $2,530 \mathrm{~cm}^{-1}$. We note that Boulard et al. ${ }^{6}$ reported a redox reaction on laser heating of $\left(\mathrm{Mg}_{0.25} \mathrm{Fe}_{0.75}\right) \mathrm{CO}_{3}$, resulting in the formation of magnetite $\left(\mathrm{Fe}_{3} \mathrm{O}_{4}\right)$ together with the post-magnesite phase. However, the new infrared bands observed at ambient pressure after decompression cannot be assigned to magnetite ${ }^{14,15}$.

First-principles calculations. To interpret the infrared spectrum measured for the post-magnesite phase, we carried out firstprinciples calculations of the infrared spectra of $\mathrm{MgCO}_{3}$ at low and high pressure, and we identified specific vibrational modes that are present only in the post-magnesite phase. Calculation were conducted for pure $\mathrm{MgCO}_{3}$ instead of the solid solution, 
a

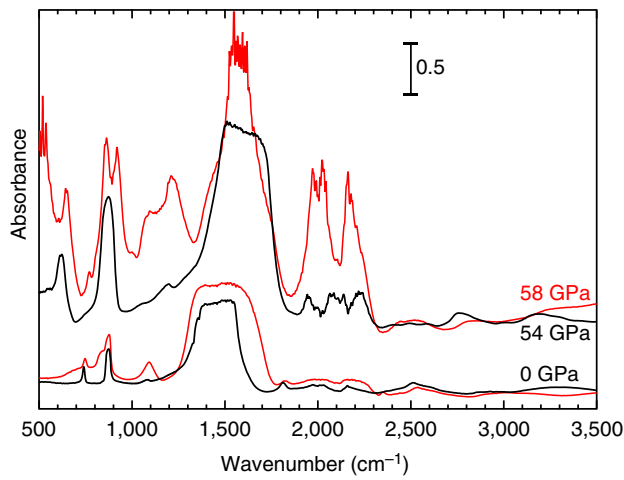

b

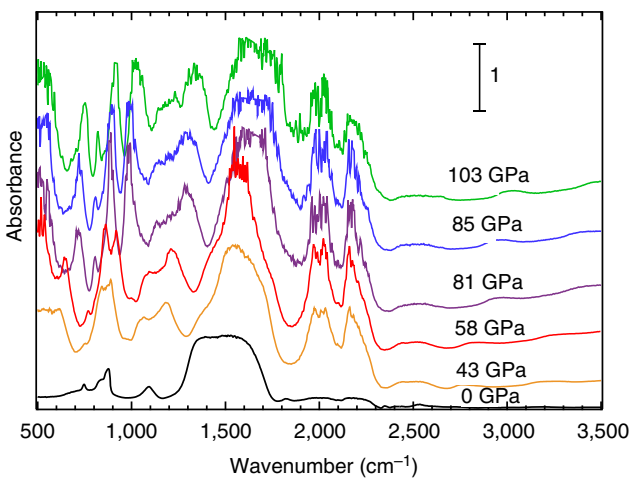

Figure 2 | In-situ infrared measurement at high pressure. (a) Experimental infrared spectra collected on compression of the ferromagnesite (black lines) at 0 and $54 \mathrm{GPa}$, and on decompression of the post-magnesite phase (red lines) at 58 and $0 \mathrm{GPa}$. The region between 1,900 and 2,300 $\mathrm{cm}^{-1}$ is dominated by absorption from the diamond anvils. (b) Experimental infrared spectra collected on the decompression of the post-magnesite phase from 103 to $0 \mathrm{GPa}$. The scale bars give the absorbance scale for each panel.

a

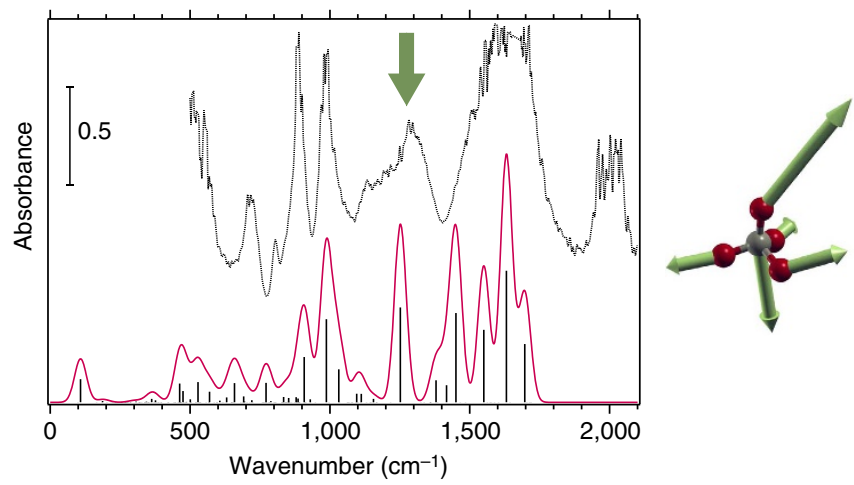

Figure 3 | Theoretical infrared results. (a) Calculated infrared intensities (black lines) and spectrum (solid red line) of the post-magnesite phase at $82 \mathrm{GPa}$. For comparison, the experimental spectrum collected at $81 \mathrm{GPa}$ is shown as a dotted line. The scale bar gives the absorbance scale. (b) The vibrational mode at $1,252 \mathrm{~cm}^{-1}$ (marked by the arrow on $\mathbf{a}$ ) is identified as a unique signature of the high-pressure phase.

$(\mathrm{Mg}, \mathrm{Fe}) \mathrm{CO}_{3}$, for computational simplicity. We used density functional theory (DFT), a semi-local exchange-correlation (xc) functional ${ }^{16}$, plane-wave basis sets and pseudopotentials. We first calculated the infrared spectrum of magnesite (symmetry group: $\mathrm{R}-3 \mathrm{C}$ ), the phase of $\mathrm{MgCO}_{3}$ stable at ambient conditions (see Supplementary Fig. 3). We used a rhombohedral cell, with the lattice constant fixed at the experimental value of $5.675 \AA$ and a cell angle of $48.2^{\circ}$ (ref. 17; Supplementary Table 1). The computed frequencies of the TO component of the $v_{2}, v_{3}$ and $v_{4}$ modes are $825,1,411$ and $725 \mathrm{~cm}^{-1}$, respectively. The theoretical frequencies computed for a single crystal are lower than the experimental ones by $\sim 4 \%$ (refs 11,18 ). This discrepancy is probably due to the use of the semi-local xc functional PerdewBurke-Ernzerhof ${ }^{16}$. By using the hybrid functional, B3LYP, Valenzano et al. ${ }^{19}$ found a smaller error of $\sim 0.5 \%$ for these three bands compared with the experimental results ${ }^{11,18}$. The infrared spectrum of the same magnesite structure computed at a pressure of $83 \mathrm{GPa}$, without allowing for any phase transition (Supplementary Table 1), shows that the TO component of the $v_{2}$ mode is weakly modified (we found a modest blue shift of $5 \mathrm{~cm}^{-1}$ ), the $v_{4}$ mode (TO) is blue-shifted to $913 \mathrm{~cm}^{-1}$ and the $v_{3}$ mode (TO) is blue-shifted to $1,647 \mathrm{~cm}^{-1}$ (Supplementary Fig. 4).
Figure 3 shows the calculated infrared spectrum of the postmagnesite phase, compared with the experimental spectrum collected at $\sim 81 \mathrm{GPa}$. We considered the structure of the postmagnesite (symmetry group: $\mathrm{C} 2 / \mathrm{m}$ ) previously measured for $\mathrm{MgCO}_{3}$ at $82 \mathrm{GPa}$ and high temperature $(\sim 2,300 \mathrm{~K})$ by Boulard et $a .^{6}{ }^{6}$, with lattice constants $a=8.39 \AA, b=6.41 \AA, c=6.82 \AA$ and $\beta=105.49^{\circ}$ (Supplementary Table 2 ). This structure is composed of tetrahedrally coordinated carbon atoms (Fig. 1b). In our computed spectrum, the phonon momentum is along the $y$ axis (crystal direction: [010]) to take into account the longitudinaloptical (LO)-TO splitting (infrared spectra obtained by the TO component and the phonon momenta along the $z$ and $x$ directions are shown in Supplementary Fig. 5). We found three bands, at 659, 771 and $1,252 \mathrm{~cm}^{-1}$, slightly red-shifted with respect to experiments $\left(\sim 715,803\right.$ and $\left.1,304 \mathrm{~cm}^{-1}\right)$, again most probably because of the approximation introduced by the semilocal functional used in our calculations (Supplementary Table 3). The two bands measured at 890 and $987 \mathrm{~cm}^{-1}$ were well reproduced by our calculations; we found 908 and $987 \mathrm{~cm}^{-1}$, respectively. Overall, the theoretical and experimental spectra were in good agreement and, most importantly, they both exhibit a band at $\sim 1,300 \mathrm{~cm}^{-1}$, which is not present in magnesite at ambient conditions or in the infrared spectrum of the isotropically compressed magnesite phase (Supplementary Fig. 4). The compressed magnesite does not exhibit any extra band between 1,000 and $1,400 \mathrm{~cm}^{-1}$, providing strong evidence that the experimental and theoretical band at $\sim 1,300 \mathrm{~cm}^{-1}$ signals the presence of a structural and bonding change.

Our geometry optimization of the tetrahedrally coordinated post-magnesite phase showed that the $\mathrm{CO}_{4}$ units, with nominal negative charge of four electrons, are asymmetric with both $\mathrm{C}$ and $\mathrm{O}$ atoms being $s p^{3}$ bonded, as shown in Fig. 4, which reports hybrid orbitals (maximally localized Wannier functions ${ }^{20}$ ) obtained from linear combinations of the eigenstates of the DFT Hamiltonian used in our calculations ${ }^{21}$. In the primitive cell of the high-pressure phase, carbon atoms have two symmetry non-equivalent positions, $\mathrm{C} 1$ and $\mathrm{C} 2$ (ref. 5). We found that for the $\mathrm{CO}_{4}$ units consisting of the $\mathrm{C} 1$ atoms, the two longer $\mathrm{C}-\mathrm{O}$ bonds are 1.427 and $1.375 \AA$, respectively, and the two shorter ones are 1.256 and $1.287 \AA$, respectively. For the other type of $\mathrm{CO}_{4}$ units ( $\mathrm{C} 2$ atoms), the two longer $\mathrm{C}-\mathrm{O}$ bonds have the same length of $1.391 \AA$, while the two shorter ones are 1.285 and $1.276 \AA$, respectively. However, the bonding structure of these two units is similar and represented in both cases by the hybrid orbitals shown in Fig. 4. The vibrational modes corresponding to the shorter $\mathrm{C}-\mathrm{O}$ bonds are at frequencies higher than 
a

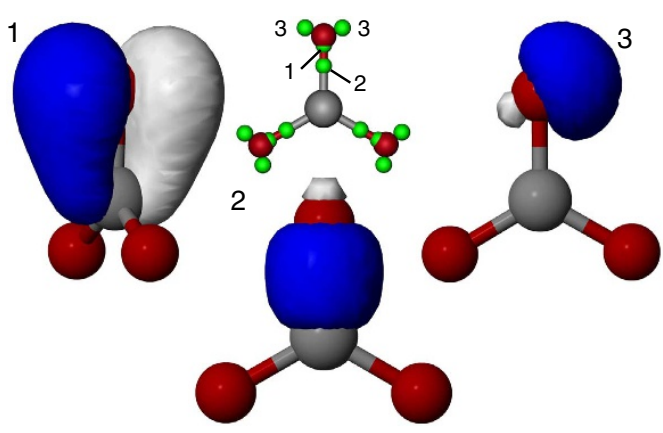

b

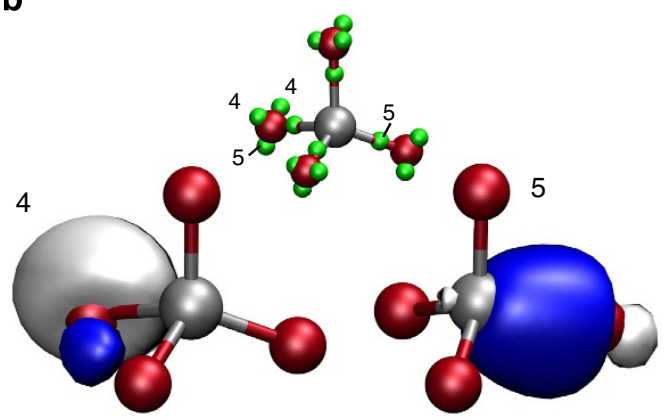

Figure 4 | Hybrid orbitals in C-O bonds. Threefold (a) and fourfold (b) coordinated carbon atoms in the magnesite and post-magnesite phases, respectively. Grey and red spheres represent $\mathrm{C}$ and $\mathrm{O}$ atoms, respectively. Green spheres represent the centres of the hybrid orbitals for the $\mathrm{CO}_{3}^{2-}$ anion (a), with 24 valence electrons and 12 doubly occupied hybrids, and for the $\mathrm{CO}_{4}^{4-}$ anion (b) with 32 valence electrons. The white and blue colours denote the positive and negative parts of the wave function, respectively. In magnesite both $\mathrm{C}$ and $\mathrm{O}$ are $s p^{2}$ bonded and three distinct orbitals were identified: $\sigma$-like orbitals localized on the $\mathrm{C}-\mathrm{O}$ bonds (orbital 2 ), $\pi$-like bonds formed by the overlap of $p_{z}$ orbitals on the $O$ and $C$ atoms (orbital 1) and lone pairs (orbitals 3 ) on the oxygen atoms; in post magnesite, both $\mathrm{C}$ and $\mathrm{O}$ are $s p^{3}$ bonded and two distinct orbitals were identified: $\sigma$-like orbitals localized on the $\mathrm{C}-\mathrm{O}$ bonds (orbital 5) and lonepair hybrids (orbitals 4) on the oxygen atoms. Hybrid orbitals were defined using maximally localized Wanier functions, built from linear combinations of single-particle electronic eigenstates (see Methods section).

$1,400 \mathrm{~cm}^{-1}$. At $81 \mathrm{GPa}$ (Figs $2 \mathrm{~b}$ and 3 ), the band at $1,452 \mathrm{~cm}^{-1}$ originates from the stretching mode of the $1.287 \AA \mathrm{C}-\mathrm{O}$ bonds and the one at $1,550 \mathrm{~cm}^{-1}$ from the stretching mode of the 1.285 and $1.276 \AA \mathrm{C}-\mathrm{O}$ bonds. Finally, the modes at 1,631 and $1,696 \mathrm{~cm}^{-1}$ are assigned to the stretching modes of the $1.256 \AA$ $\mathrm{C}-\mathrm{O}$ bonds. We note that in the experimental spectra, the bands between 1,400 and $1,700 \mathrm{~cm}^{-1}$ are saturated and individual modes cannot be identified. The vibrational mode at $1,252 \mathrm{~cm}^{-1}$ originates from the stretching mode of the 1.375 and $1.391 \AA$ $\mathrm{C}-\mathrm{O}$ bonds, with the phonon momentum along the $y$ axis (Fig. 3b). The TO component of this mode is expected at $1,155 \mathrm{~cm}^{-1}$. The bands between 1,100 and $1,300 \mathrm{~cm}^{-1}$ may stem from the different LO and TO components of the $1,252 \mathrm{~cm}^{-1}$ stretching mode found in the experimental spectrum. As shown in Supplementary Fig. 5, when the phonon momentum is along the $x$ or $z$ directions, the frequency of the stretching mode of the longer $\mathrm{C}-\mathrm{O}$ bonds is lower than $1,252 \mathrm{~cm}^{-1}$. The intensities of the LO and TO components in powder transmission spectra depend on the size and shape of the powder particles. There is an apparent band at $1,304 \mathrm{~cm}^{-1}$ in the experimental spectrum, suggesting that the thickness of many sample particles on the $y$ direction may be much less than the wavelength of exciting radiation $(7.7 \mu \mathrm{m} \text { in air })^{22}$.

The modes detected between 1,100 and $1,300 \mathrm{~cm}^{-1}$ are at lower frequencies than the asymmetric stretching mode of the planar carbonate ion $v_{3}$ measured at ambient conditions $\left(1,401 \mathrm{~cm}^{-1}\right.$ in this study). This is consistent with $\mathrm{C}-\mathrm{O}$ bond lengths of $1.375 \sim 1.391 \AA$ found in the $\mathrm{CO}_{4}$ units, which are longer than that of the carbonate ion present in magnesite at ambient conditions ( $\sim 1.30 \AA$ ) $)$. As expected, a longer bond length corresponds to a lower vibrational frequency. Such a trend was also found in magnesite by Grzechnik et al. ${ }^{23}$, who observed a shift of the $v_{3}$ mode to lower frequency by $\sim 114 \mathrm{~cm}^{-1}$ when decompressing the sample from $29 \mathrm{GPa}$ to ambient conditions. Consistently, our calculation showed that when releasing the pressure of magnesite, from 58 to $0 \mathrm{GPa}$, the $\mathrm{C}-\mathrm{O}$ bonds increase by $0.04 \AA$, and thus the TO component of the $v_{3}$ mode redshifts by $194 \mathrm{~cm}^{-1}$.

\section{Discussion}

In summary, we report the first in situ characterization of $\mathrm{C}-\mathrm{O}$ bonds of the post-magnesite phase. We found that at high pressure, on transformation into the post-magnesite phase, the infrared spectrum of $(\mathrm{Mg}, \mathrm{Fe}) \mathrm{CO}_{3}$ exhibits novel, unique features not present in the low-pressure spectrum, which we assigned to a fundamentally different bonding configurations of carbon. Carbon bonds transform from $s p^{2}$ (in the trigonal, doubly charged carbonate anions) to $s p^{3}$ hybridized configurations (in the tetrahedral tetra-charged carbonate anions), which we characterized using $a b$ initio calculations within DFT. Hence, our study provides the identification of a mode at $1,304 \mathrm{~cm}^{-1}$, which may be used as a fingerprint of tetrahedrally bonded carbon in high-pressure mineral phases. Carbon tetrahedrally bonded to oxygen has also been proposed to be present in high-pressure phase- $\mathrm{V}$ of $\mathrm{CO}_{2}$ (refs 24,25). However, in this phase, tetrahedral $\mathrm{CO}_{4}$ groups are symmetric with a C-O distance of $1.35 \AA$, similar to the longer $\mathrm{C}-\mathrm{O}$ distances observed here. Measured infrared spectra showed the presence of a mode in the same frequency range at $1,126 \mathrm{~cm}^{-1}$; however, all modes were reported to involve simultaneous stretching and bending, unlike the pure stretching mode $^{24}$ identified here.

The dramatic change we found in the carbon environment in ferromagnesite may have significant implications on carbon reservoirs and fluxes in the lower mantle and, therefore, on the deep carbon cycle. For example, the new bonding configuration, identified here in the high-pressure carbonate structure, is dramatically different from trigonal planar group in the ambient conditions structure. This will probably influence its chemical and physical properties such as its reactivity. Moreover, one would expect dramatic changes in the behaviour of carbonate melts with increases in coordination of carbon, owing to the ability of $\mathrm{CO}_{4}$ to form polymerizable networks while $\mathrm{CO}_{3}$ trigonal groups can not $^{26}$. At upper mantle conditions, carbonate melts differ from silicate melts. They exhibit ultra-low viscosity potentially resulting in high mobilities ${ }^{27}$. Preliminary theoretical studies predict that carbonate melt viscosity increases at high pressures ${ }^{28}$, which would inhibit mobility of carbonate melts in the lower mantle and might lead to the presence of deep carbon reservoirs.

\section{Methods}

In situ high-pressure infrared spectroscopy. The infrared absorbance was measured in the $500-4,000 \mathrm{~cm}^{-1}$ range through a symmetric Mao-type DAC mounted with type-IIa diamond anvils. Measurements were conducted with a Bruker Vertex $80 / \mathrm{v}$ spectrometer at the side station of the high-pressure infrared beamline U2A of the National Synchrotron Light Source. The spectral resolution was $4 \mathrm{~cm}^{-1}$ and the beam size under the microscope was $\sim 20 \times 20 \mu \mathrm{m}$.

Absorption infrared spectra were obtained after normalization against a background spectrum collected on an area with $\mathrm{KBr}$ pressure medium only (set 1 ) at 
each pressure or in the empty DAC (set 2) at ambient pressure. A natural sample of $\left(\mathrm{Mg}_{0.25} \mathrm{Fe}_{0.75}\right) \mathrm{CO}_{3}$ siderite, provided by the mineralogical collection of University of Pierre et Marie Curie, was used in this experiment. For both sets of experiments, powdered $\left(\mathrm{Mg}_{0.25} \mathrm{Fe}_{0.75}\right) \mathrm{CO}_{3}$ was loaded together with a ruby ball and the pressure was determined before and after infrared absorbance data collection from the shift of the ruby fluorescence line ${ }^{29}$. In the first set of experiments, $\mathrm{KBr}$ was added as a pressure-transmitting medium in a DAC with $400 \mu \mathrm{m}$ culets. One run was performed with a sufficiently thin sample, to obtain good quality of the highintensity band $v_{3}$, and a second run with a thicker sample, to get good data quality of the lower-intensity bands. In the second set of experiments, the sample was loaded without pressure medium in a DAC with $70 \mu \mathrm{m}$ culets and compressed up to $103 \mathrm{GPa}$. The sample was then transformed into the post-magnesite phase by laser heating at $\sim 2,100 \mathrm{~K}$ at HPCAT, Argonne National Laboratory. We used in-situ XRD to monitor the transformation into the post-magnesite phase

DFT calculations. DFT calculations were performed with the Quantum ESPRESSO package ${ }^{30}$ and maximally localized Wannier functions were computed with the Qbox code (v.1.56.2, http://eslab.ucdavis.edu/software/qbox/) $)^{31}$ and the algorithm of reference ${ }^{21}$. We used the Perdew-Burke-Ernzerhof $x c$ functional ${ }^{16}$ plane-wave basis sets and ultrasoft pseudopotentials ${ }^{32}$. We used a kinetic energy cutoff of $40 \mathrm{Ry}$ for wavefunctions and $360 \mathrm{Ry}$ for charge densities. Our structural optimizations were performed by fully relaxing all atomic coordinates within the primitive cell until the force on each atom was smaller than $10^{-4} \mathrm{Ry} \mathrm{Bohr}^{-1}$. We used $3 \times 3 \times 3 k$-point mesh for the primitive cell of magnesite and $2 \times 2 \times 2$ $k$-point mesh for the post-magnesite phase. We used the unit cell and atomic positions of the postmagnesite reported in Boulard et al. ${ }^{6}$ in which the space group is $\mathrm{P} 21 / \mathrm{c}$ after transferring the atomic positions from $\mathrm{C} 2 / \mathrm{m}$ as reported in Oganov et al. ${ }^{5}$ to P21/c. In the DFT calculations, the point group $2 / \mathrm{m}$ was used.

We carried out phonon calculations using density functional perturbation theory $^{33}$ to obtain the infrared spectra. The intensity of the $m$ th infrared mode is defined $\mathrm{as}^{34}$ :

$$
I_{m}=\sum_{\alpha}\left|\sum_{s \beta} Z_{\alpha \beta}^{*}(s) e_{m}(s, \beta)\right|^{2}
$$

where $\alpha$ and $\beta$ are Cartesian components, $Z_{\alpha \beta}^{*}(s)$ is the Born effective charge tensor of the sth atom and $e_{m}(s, \beta)$ is the normal mode eigenvector ${ }^{34}$. The calculated intensities were broadened by a Gaussian function with the full width of $50 \mathrm{~cm}^{-1}$ at half maximum.

\section{References}

1. Wood, B. J., Pawley, A. \& Frost, D. R. Water and carbon in the Earth's mantle. Philos. Trans. R. Soc. London 354, 1495-1511 (1996).

2. Isshiki, M. et al. Stability of magnesite and its high-pressure form in the lowermost mantle. Nature 427, 60-63 (2004).

3. Santillán, J. \& Williams, Q. A high-pressure infrared and X-ray study of $\mathrm{FeCO}_{3}$ and $\mathrm{MnCO}_{3}$ : comparison with $\mathrm{CaMg}\left(\mathrm{CO}_{3}\right)_{2}$-dolomite. Phys. Earth Planet. Inter 143-144, 291-304 (2004).

4. Oganov, A. R., Glass, C. W. \& Ono, S. High-pressure phases of $\mathrm{CaCO}_{3}$ : Crystal structure prediction and experiment. Earth Planet. Sci. Lett. 241, 95-103 (2006).

5. Oganov, A. R., Ono, S., Ma, Y., Glass, C. W. \& Garcia, A. Novel high-pressure structures of $\mathrm{MgCO}_{3}, \mathrm{CaCO}_{3}$ and $\mathrm{CO}_{2}$ and their role in Earth's lower mantle. Earth Planet. Sci. Lett. 273, 38-47 (2008).

6. Boulard, E. et al. New host for carbon in the deep Earth. Proc. Natl Acad. Sci. USA 108, 5184-5187 (2011)

7. Boulard, E. et al. Experimental investigation of the stability of Fe-rich carbonates in the lower mantle. J. Geophys. Res. 117, B02208 (2012).

8. Tao, R., Fei, Y. \& Zhang, L. Experimental determination of siderite stability at high pressure. Am. Mineral. 98, 1565-1572 (2013).

9. Lavina, B. et al. Siderite at lower mantle conditions and the effects of the pressure-induced spin-pairing transition. Geophys. Res. Lett. 36, L23306 (2009).

10. Farfan, G. et al. Bonding and structural changes in siderite at high pressure. Am. Mineral. 97, 1421-1426 (2012).

11. Huang, C. K. \& Kerr, P. F. Infrared study of the carbonate minerals. Am. Mineral. 45, 311-324 (1960).

12. Lane, M. D. \& Christensen, P. R. Thermal infrared emission spectroscopy of anhydrous carbonates. J. Geophys. Res. 102, 25,581-25,592 (1997).

13. Gunasekaran, S., Anbalagan, G. \& Pandi, S. Raman and infrared spectra of carbonates of calcite structure. J. Raman Spectrosc. 37, 892-899 (2006).

14. Chamritski, I. \& Burns, G. Infrared- and Raman-active phonons of magnetite, maghemite, and hematite: a computer simulation and spectroscopic study. J. Phys. Chem. B 109, 4965-4968 (2005).

15. Gasparov, L. et al. Infrared and Raman studies of the Verwey transition in magnetite. Phys. Rev. B 62, 7939-7944 (2000).
16. Perdew, J., Burke, K. \& Ernzerhof, M. Generalized Gradient Approximation Made Simple. Phys. Rev. Lett. 77, 3865-3868 (1996).

17. Graf, D. L. Crystallographic tables for the rhombehedral carbonates. Am. Mineral. 46, 1283-1316 (1961)

18. Hellwege, K. H., Lesch, W., Plihal, M. \& Schaack, G. Zwei-phononenabsorptionsspektren und dispersion der schwingungszweige in kristallen der kalkspatstruktur. Zeitschrift Phys. 232, 61-86 (1970).

19. Valenzano, L. et al. Ab initio vibrational spectra and dielectric properties of carbonates: magnesite, calcite and dolomite. Theor. Chem. Acc. 117, 991-1000 (2007).

20. Marzari, N., Mostofi, A. a., Yates, J. R., Souza, I. \& Vanderbilt, D. Maximally localized Wannier functions: theory and applications. Rev. Mod. Phys. 84, 1419-1475 (2012).

21. Gygi, F., Fattebert, J.-L. \& Schwegler, E. Computation of maximally localized Wannier functions using a simultaneous diagonalization algorithm. Comput. Phys. Commun. 155, 1-6 (2003).

22. Farmer, V. C. Differing effects of particle size and shape in the infrared and Raman spectra of kaolinite. Clay Miner. 33, 601-604 (1998).

23. Grzechnik, A., Simon, P., Gillet, P. \& McMillan, P. An infrared study of $\mathrm{MgCO}_{3}$ at high pressure. Phys. B 262, 67-73 (1999).

24. Santoro, M. et al. Partially collapsed cristobalite structure in the non molecular phase $\mathrm{V}$ in $\mathrm{CO}_{2}$. Proc. Natl Acad. Sci. USA 109, 5176-5179 (2012).

25. Datchi, F., Mallick, B., Salamat, A. \& Ninet, S. Structure of polymeric carbon dioxide $\mathrm{CO}_{2}$-V. Phys. Rev. Lett. 108, 125701 (2012).

26. Jones, A. P., Genge, M. \& Carmody, L. Carbonate melts and carbonatites. Rev. Mineral. Geochem. 75, 289-322 (2013).

27. Kono, Y. et al. Ultralow viscosity of carbonate melts at high pressures. Nat. Commun. 5, 5091 (2014).

28. Jones, A. P., Genge, M. \& Carmody, L. Carbonate Melts and Carbonatites. Rev Mineral Geochem. 75, 289-322 (2013).

29. Mao, H. K., Xu, J. \& Bell, P. M. Calibration of the Ruby pressure gauge to 800 kbar under quasi-hydrostatic conditions. J. Geophys. Res. 91, 4673-4676 (1986).

30. Giannozzi, P. et al. QUANTUM ESPRESSO: a modular and open-source software project for quantum simulations of materials. J. Phys. Condens. Matter 21, 395502 (2009).

31. Gygi, F. Architecture of Qbox: a scalable molecular dynamics code. IBM J. Res. Dev. 52, 1-8 (2008).

32. Vanderbilt, D. Soft self-consistent pseudopotentials in generalized eigenvalue formalism. Phys. Rev. B 41, 7892-7895 (1990).

33. Baroni, S., de Gironcoli, S., dal Corso, A. \& Giannozzi, P. Phonons and related crystal properties from density-functional perturbation theory. Rev. Mod. Phys. 73, 515-562 (2001).

34. Giannozzi, P. \& Baroni, S. Vibrational and dielectric properties of C60 from density-functional perturbation theory. J. Chem. Phys. 100, 8537 (1994).

\section{Acknowledgements}

The use of the U2A beamline was supported by COMPRES, the Consortium for Materials Properties Research in Earth Sciences under NSF Cooperative Agreement EAR 11-57758 and the DOE-NNSA (DE-FC03-03N00144, CDAC). The use of National Synchrotron Light Source was supported by the US Department of Energy (DOE), Office of Science, Office of Basic Energy Sciences (BES), under contract number DE-AC0298CH10886. In-situ laser heating and XRD were performed at the Argonne National Laboratory HPCAT (Sector 16), which is supported by DOE-NNSA, DOE-BES and NSF A.P.S. is supported by DOE-BES, under contract number DE-AC02-06CH11357. We thank C.Y. Shi for help with the sample preparation. E.B. and D.P. acknowledge support from the Deep Carbon Observatory. G.G. acknowledges support from DOE/BES DE-SC0008938. W.L.M. acknowledges support from NSF, Geophysics Grant EAR-1141929.

\section{Author contributions}

E.B. designed the study. E.B. and Z.L. conducted the experiments. E.B. analysed the data. D.P. performed the theoretical calculations. E.B. and D.P. wrote the manuscript. All authors discussed the results and commented on the manuscript.

\section{Additional information}

Supplementary Information accompanies this paper at http://www.nature.com/ naturecommunications

Competing financial interests: The authors declare no competing financial interest.

Reprints and permission information is available online at http://npg.nature.com/ reprintsandpermissions/

How to cite this article: Boulard, E. et al. Tetrahedrally coordinated carbonates in Earth's lower mantle. Nat. Commun. 6:6311 doi: 10.1038/ncomms7311 (2015). 